\title{
Aprendizajes reales en ambientes virtuales: El rol de la tecnología en la era de la Inteligencia Artificial y el Big Data
}

\author{
Real learnings in virtual environments: \\ The role of technology in the Artificial Intelligence and Big Data era
}

Recibido: 18 de marzo de 2020 / Aceptado: 18 de mayo de 2020

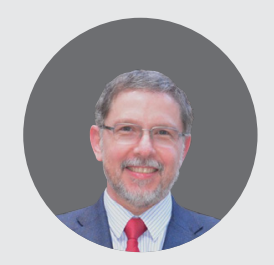

URIEL CUKIERMAN

UNIVERSIDAD TECNOLÓGICA

NACIONAL (UTN) DE ARGENTINA

uriel@cukierman.name

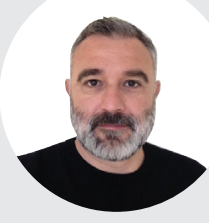

EDUARDO VENDRELL VIDAL

UNIVERSITAT POLITĖCNICA DE VALÈNCIA even@upv.es

\section{Resumen}

La Inteligencia Artificial y el Big Data han cobrado relevancia en los ámbitos académicos recientemente y se nos plantean interrogantes sobre su aplicación y utilidad. En este artículo analizaremos los significados de estas tecnologías, las implicancias de su utilización en el ámbito educativo y el impacto que pudieran tener en la labor pedagógica. Lo haremos a partir de los cuestionamientos más habituales que toda nueva tecnología presenta cuando se la pretende utilizar en educación y con base en una sucinta investigación bibliográfica que le dé sentido y sustento a este abordaje, concluyendo así en lo que sabemos desde hace mucho, y es que la tecnología no vale por sí misma, sino por el uso que hacemos de ella.

Palabras clave: Inteligencia Artificial; Big Data; Analíticas de aprendizaje; Aprendizaje adaptativo.

\section{Abstract}

Artificial Intelligence and Big Data have recently gained relevance in academic fields, raising questions about their application and usefulness. In this article we will analyze the meanings of these technologies and the implications of their use for pedagogical purposes. We will do so based on the most frequent questions that every new technology presents when it is intended to be used in education, and based on a bibliographic research that gives meaning and support to this approach, concluding in what we have known for long time now, and it is that technology is not worth by itself, but by the use we make of it.

Keywords: Artificial Intelligence; Big Data; Learning analytics; Adaptive learning. a. Master en Tecnologías de la Información Aplicadas a la Empresa por la Universidad Politécnica de Madrid e Ingeniero Electrónico por la Universidad Tecnológica
Nacional (UTN) de Argentina. Actualmente es profesor y director del Centro de Investigación e Innovación Educativa en la UTN. Para contactar al autor: uriel@cukierman.name

b. Doctor en Informática por la Universidad Politécnica de Valencia. Actualmente es profesor y Vicerrector de Estudios, Calidad y Acreditación en dicha Universidad. Para contactar al autor: even@upv.es 


\section{Introducción}

Muchos podrían creer que la tecnología es algo que surgió en el siglo $\mathrm{XX}$ y que ha alcanzado su mayor impacto en este siglo $\mathrm{XXI}$, pero no es cierto, desde siempre ha existido tecnología. Algunos todavía hoy se sienten escépticos con ella, a otros les apasiona. En el plano de la Educación, unos enseñan Tecnología a nivel escolar como una asignatura del currículo; otros, a nivel universitario, son los responsables de conducir a la nueva generación en los avances tecnológicos en las carreras relacionadas con la informática. Sin embargo, hoy todos los docentes debemos revestirnos de una nueva competencia, no importa si nos gusta o no la tecnología, si somos o no somos del área, es imprescindible que nos aboquemos todos a la Tecnología Educativa.

Muchos hemos avanzado ya un largo recorrido de aprendizaje, investigación y experimentación en el campo que nos ha llevado desde el desarrollo de plataformas educativas en línea (Cukierman, 2005), hasta la implementación de redes de videoconferencia (Castro, Meier, Cukierman, Waigandt, 2015), pasando por las más variadas experiencias educativas con mayor o menor incorporación de recursos tecnológicos (Cukierman et al., 2017 y 2018). En estos tiempos, la tecnología es cada vez más omnipresente y ubicua. Las discusiones entre tecnofóbicos y tecnofílicos ya son cosa del pasado. Hoy sabemos que la cuestión no es la tecnología en sí misma, sino cómo y para qué se usa. Primero, la discusión fue si se permitía el uso de calculadoras electrónicas, luego, el objeto controversial fueron los celulares y ahora podrían serlo la Inteligencia Artificial (IA) y el Big Data (BD).

Este artículo se detiene en estos dos últimos conceptos y pretende analizar su significado, su posible uso en el ámbito educativo y el impacto que pueden tener en la labor pedagógica.

En primer lugar, se hará un somero repaso a los conceptos de IA y BD, su origen y su desarrollo básico inicial, para luego analizar su aplicación al ámbito educativo, considerando si su uso en este entorno puede aportar una mejora en el aprendizaje de los estudiantes. Este análisis se aborda desde la consideración de aplicaciones concretas y deteniéndonos en unos de los aspectos clave del aprendizaje: la evaluación. Finalmente, se emiten unas conclusiones como resultado del análisis.

\section{Una primera aproximación conceptual al tema}

Es muy común que se hable de educación virtual cuando se refiere a educación a distancia o mediada por tecnología. Por eso es oportuno iniciar este estudio haciendo algunas reflexiones conceptuales al respecto. Virtual refiere a algo opuesto a lo real, por lo tanto, algo que no existe. Por eso sería preferible no utilizar el término virtual para adjetivar a la educación. Lo que importa en educación es lo que el alumno realmente aprende que, es importante aclarar, no es lo mismo que lo que el profesor enseña. Por eso nos centraremos en los aprendizajes reales que ocurren en espacios que no tienen existencia física palpable, entonces diremos que dichos aprendizajes se desarrollan en ambientes virtuales, los cuales son mediados por recursos tecnológicos.

Ahora bien, ya en esta segunda década del siglo XXI la IA y el BD están en boca de todos, quizás con poco conocimiento del significado de estos conceptos y con mucho de fantasía generada por novelas, películas y muchos "opinadores" que hablan del tema sin molestarse en indagar en búsqueda de información seria al respecto. Por ello es oportuno en este punto dedicar algunas líneas a definir ambos conceptos de una manera precisa. Según la Real Academia de la lengua española, la Inteligencia Artificial, en lo sucesivo IA, es un concepto que refiere a la "disciplina científica que se ocupa de crear programas informáticos que ejecutan operaciones comparables a las que realiza la mente humana, como el 
aprendizaje o el razonamiento lógico" (Real Academia Española, s.f.). El concepto no es realmente nuevo, de hecho fue en el año 1955 que aparece mencionado por primera vez en un trabajo que es considerado la piedra basal de este nuevo campo. En este trabajo, los autores se refieren a la IA diciendo que:

Se intentará encontrar cómo hacer que las máquinas utilicen el lenguaje, formen abstracciones y conceptos, resuelvan tipos de problemas que ahora están reservados para los humanos y se mejoren a sí mismos. [...] Para el propósito actual, se considera que el problema de la inteligencia artificial consiste en hacer que una máquina se comporte de una manera que se llamaría inteligente si un humano se comportara de esa manera. (McCarthy, Minsky, Rochester, y Shannon, 1955)

Vayamos ahora pues por la definición del otro concepto involucrado en este estudio, o sea, el de Big Data, en lo sucesivo BD. Si bien en algunos textos en español se suele referir a este concepto como "Datos Masivos", es más común hablar de él utilizando su denominación en inglés, idioma en el cual se lo considera un sustantivo, por ello es que nos referiremos a "el" BD. El manejo de grandes cantidades de datos se remonta a épocas muy lejanas en el tiempo, pero es recién en los últimos años que se ha producido un crecimiento exponencial del volumen de información disponible y apta para ser procesada. Este aumento exponencial de los datos tiene relación directa, obviamente, con el desarrollo de la tecnología y de su amplio uso. No obstante, hay un fenómeno o desarrollo intrínsecamente asociado al BD: la accesibilidad a la información y la conectividad. Con ello han proliferado las aplicaciones, algoritmos y dispositivos físicos que permiten conectarse a Internet y entre ellos mismos, generando posibilidades no imaginadas hasta hace bien poco y ofreciendo oportunidades de desarrollo en ámbitos muy diferentes. El Internet de las Cosas (Internet of Things, IoT), es un efecto derivado de esta conectividad y un factor clave en la proliferación de los datos. La conectividad entre dispositivos $y$ entre humanos $y$ dispositivos genera una cantidad de datos considerable, nunca antes imaginada, que supone una oportunidad en lo que respecta a su análisis y la toma de decisiones asociada.

Se atribuye a Roger Magoulas, de la editorial O’Reilly, la primera utilización del término BD en un artículo del año 2005 (Halevi y Moed, 2012). Ahora bien, la acumulación de muchos datos no es necesariamente un sinónimo de BD. En realidad, el $B D$ se trata de una forma de recopilar datos "crudos" de múltiples y diversas fuentes, almacenarlos para ser procesados por los programas de análisis y usarlos para derivar valor y significado en formas completamente nuevas (Perry, 2017). Se suele hablar de las " 4 V" del BD, volumen, variedad, velocidad y veracidad.

Hechas estas aclaraciones conceptuales, en la próxima sección trataremos de relacionar estos conceptos con la educación.

\section{3. ¿Qué relación tienen la IA y el BD con la educación?}

A primera vista pareciera que estos conceptos no tienen nada que ver con la educación, en la que tratamos con inteligencias humanas $\mathrm{y}$, por lo tanto, absolutamente reales $\mathrm{y}$, en general, con datos muy personalizados, o por lo menos así pretendemos que sea. En efecto, cuando hablamos del aprendizaje que queremos que alcancen nuestros alumnos, lo asociamos con el desarrollo de sus inteligencias, dicho así, en plural, tal como lo propuso Gardner en su ya famosa "Teoría de las inteligencias múltiples" (Gardner, 1983) (Gardner y Hatch, 1989) (Gardner, 1991), que no tienen nada de artificial.

No obstante, la tecnología se ha hecho imprescindible en todos los contextos, y así vemos que la IA y el BD están generando 
uno de los mercados de mayor crecimiento en la educación (Technavio, 2018). Obviamente, que haya un mercado no implica que lo que se vende sea bueno, sino que hay alguien dispuesto a comprarlo. Pero si hay tanto interés en invertir ingentes sumas de dinero en este tipo de tecnología, eso nos lleva a preguntarnos sobre su utilidad y, para responder esta pregunta, primero hay que entender cómo la IA y el BD pueden aportar a la mejora del aprendizaje.

Comencemos explorando dos conceptos directamente relacionados entre sí. Se trata de "Analíticas de Aprendizaje" y "Aprendizaje Adaptativo". El primero de ellos deriva directamente de lo que se llama "Inteligencia de Negocios" y también de lo que se conoce como "Analíticas Web", en ambos casos se trata de recolectar y analizar grandes volúmenes de información para facilitar la toma de decisiones y/o comprender el comportamiento de los usuarios (Grigori, D., Casati, F., Castellanos, M., Dayal, U., Sayal, M., y Shan, M. 2004) (Web Analytics Association, 2007). En consecuencia, podemos decir que las Analíticas de Aprendizaje constituyen una aplicación educativa de Analíticas Web que pretende obtener un perfil de los estudiantes, con base en un proceso de recopilación y análisis de las interacciones de los estudiantes con base en sus actividades de aprendizaje en línea (New Media Consortium, 2016), o sea, esto supone que todo lo que hace el estudiante lo hace a través de la Web, lo que de por sí constituye una aseveración de dudosa validez, al menos en nuestro ámbito nacional y regional (Argentina en particular y Latinoamérica en general), pero dejaremos el análisis crítico de este tema para más adelante.

Analicemos a continuación el concepto de "Aprendizaje Adaptativo" que se define como el conjunto de tecnologías que responden a las interacciones de un estudiante en tiempo real proporcionándole un apoyo individual automatizado (EdSurge, 2016). Se trata, pues, de que el modelo de aprendizaje se adapte o acondicione a las circunstancias y avance del estudiante, de manera personalizada. Esto se consigue controlando la información que se "libera" hacia el estudiante, su ritmo de aprendizaje y a través de una retroalimentación adecuada e inmediata. Estos tres elementos clave pueden ser controlados, precisamente, con la aplicación de la IA y el BD en el aprendizaje del estudiante.

A esta altura queda suficientemente clara la respuesta a la pregunta con la que iniciamos esta sección. Vayamos entonces a realizar un análisis crítico de estas tecnologías y de su impacto efectivo en los ámbitos educativos y lo haremos partiendo, nuevamente, de una pregunta.

\section{4. ¿Se pueden mejorar los aprendizajes de nuestros alumnos por medio de estas tecnologías?}

En la sección anterior ha quedado claro que la potencial efectividad de estas tecnologías está basada en la suposición que los estudiantes realizan todas o la mayoría de sus actividades a través de recursos digitales conectados en red. Hoy en día más de la mitad de la población mundial tiene acceso a Internet (International Telecommunications Union, 2019) y es por eso que empresas globales como Google y Facebook tienen su razón de ser en los datos que colectan de los miles de millones de usuarios que utilizan sus servicios. Estas y otras empresas basan sus estrategias comerciales en el análisis de la enorme cantidad de datos que obtienen gracias a los miles de millones de usuarios que buscamos información en Google o publicamos nuestras novedades en Facebook. Para ellas, la IA y el BD, y las técnicas derivadas de Inteligencia de Negocios y Analíticas Web, resultan vitales, sin estas técnicas, aquellas empresas no existirían. Pero ¿se puede transpolar esta efectividad en la determinación de nuestras necesidades como consumidores, y la consecuente satisfacción de ellas, al campo educativo y, más aún, suponer que ello permitirá mejorar los aprendizajes de los 
alumnos? Antes de sacar conclusiones apresuradas, analicemos un poco más a fondo el tema.

Es cierto que existe una tendencia creciente en la educación hacia la utilización de recursos digitales en red, y más específicamente, accesibles por Internet. Veamos algunos números para saber en qué se basa esta afirmación. En Europa, en promedio, el $73 \%$ de los estudiantes de nivel secundario superior (15 a 18 años de edad) utilizan Internet en la escuela con objetivos de aprendizaje, al menos una vez a la semana, y $65 \%$ de las escuelas en el mismo nivel utilizan un Entorno Virtual de Enseñanza y Aprendizaje (EVEA), también conocido por sus siglas en inglés LMS (Learning Management System) o, más comúnmente, Campus Virtual ${ }^{1}$ (Deloitte Ipsos MORI, 2019). En Latinoamérica la situación no es muy distinta, ya que el $75 \%$ de los países relevados cuentan con instituciones educativas primarias $y$ secundarias con instrucción asistida por Internet $^{2}$ (UNESCO, 2012). Estas estadísticas nos indican que los datos que generan nuestros alumnos cuando realizan actividades educativas a través de sistemas digitales conectados a Internet están allí y, de hecho, deben estar siendo escrutados por las mismas empresas mencionadas anteriormente. Pero volviendo a la pregunta con la que iniciamos esta sección, ¿significa esto que esos datos pueden ser utilizados para mejorar los aprendizajes? Eso es lo que nos proponen las aplicaciones de IA que a tal efecto ofrecen empresas tales como Knewton ${ }^{3}$, Clever ${ }^{4}$, Yet Analytics ${ }^{5}$ y otras. Como con cualquier otra innovación tecnológica aplicada a la educación, están sus promotores y sus detractores (El Alfy, Marx Gómez, y Dani, 2018) (Nunn, Avella, Kanai, y Kebritchi, 2016), pero la mejor forma de saber a ciencia cierta si un determinado recurso es útil en un determinado nivel educativo, disciplina, curso, etc., es mediante la investigación y experimentación en esas condiciones dadas $o$, dicho de otra manera, lo que le funciona al profesor $X$ en su curso, disciplina, institución, podría no resultarle productivo al profesor $Y$ en su propio curso, disciplina $o$ institución.

Dicho esto, analicemos en detalle las aplicaciones concretas y particulares de estas tecnologías en el ámbito educativo. El objetivo más comúnmente mencionado es el de promover un aprendizaje personalizado a partir de las "huellas digitales" que dejan los alumnos al utilizar los sistemas informáticos; adecuar sus trayectos formativos, actividades de aprendizaje, evaluaciones, etc. a sus necesidades particulares y, en un nivel más avanzado, prever su desempeño a futuro aun antes que se produzcan los resultados concretos. El objetivo pretendido es ambicioso, aunque habría que analizar si los resultados concretos justifican la inversión en tiempo y dinero que estos recursos requieren porque, justo es decirlo, no son fáciles de utilizar ni son baratos, por lo que probablemente su uso se encuentre restringido a instituciones privadas que manejen importantes recursos económicos. $Y$ aquí aparece la que es una de las principales prevenciones al respecto, ¿lo utilizarán estas instituciones para mejorar los aprendizajes de los alumnos o para fidelizar y evitar el abandono de quienes proveen los recursos económicos que solventan el funcionamiento de dichas instituciones, o sea, sus alumnos?

Analicemos a continuación otras de las aplicaciones de esta tecnología, nos referimos a los sistemas de tutoría automatizada, para decirlo más sencillamente, un asistente basado en IA que responderá las consultas de los alumnos y los orientará con sus dudas y necesidades. Algunos de nosotros ya hemos experimentado este tipo de asistentes en muchos sitios web de 
banca hogareña o de compras por Internet; se nos ofrece una asistencia que pareciera ser humana, tiene una cara y un nombre, a la que le podemos hacer preguntas pero, a poco de avanzar, nos damos cuenta que solo puede responder cuestiones bastante reguladas y que si nuestras dudas son un poco más complejas, o se salen del "libreto", nos indicará muy amablemente (eso sí, estos asistentes nunca se enojan) que un ejecutivo de cuentas se estará comunicando con nosotros en breve. $Y$ entonces podemos seguir haciéndonos preguntas, ¿es esta la personalización que pretendemos para la formación de nuestros alumnos, o será una forma de reducir personal 0 de poder atender a más personas en menos tiempo?

Veamos por lo menos una más de las pretendidas aplicaciones de esta tecnología: la evaluación. La evaluación es uno de los elementos clave en el aprendizaje de los estudiantes, por lo que resulta de especial interés el análisis del uso de la IA y el BD en este aspecto. En principio, la idea que subyace es que estos sistemas permitirían optimizar y volver más imparcial el proceso de evaluación de los aprendizajes de los alumnos. Para poder analizar las supuestas ventajas de este tipo de aplicación, debemos tener siempre presente que el objetivo de las evaluaciones (que no deben confundirse con las calificaciones que resultan de dichas evaluaciones) es servir para la mejora, mostrándole al alumno los aspectos de su aprendizaje que evolucionan favorablemente y aquellos en los que debe seguir trabajando. Está claro que dichas evaluaciones "automatizadas" pueden funcionar de manera apropiada cuando estén correctamente parametrizadas, como es el caso del examen de opciones múltiples. No obstante, en principio resultarían poco apropiadas cuando lo que se intenta evaluar son competencias, entendidas como la adecuada combinación de conocimientos, habilidades, actitudes y aptitudes. Por ejemplo, ¿cómo podría un sistema de IA evaluar la participación de un alumno en clase, o la resolución de un problema abierto o la presentación de un tema en clase, etc.?

El análisis que nos ocupa se focaliza en determinar, o al menos presumir, si la IA y el BD servirán para mejorar los aprendizajes. $Y$ para responder esta interrogante proponemos tres escenarios posibles: el de la educación tradicional enteramente presencial, el de la llamada educación híbrida o "blended learning" en la cual se combinan actividades desarrolladas de manera presencial y otras desarrolladas en línea soportadas por plataformas informáticas como las mencionadas anteriormente en este artículo y, finalmente, la educación que se realiza enteramente a distancia y, en muchos casos, con poca o ninguna interacción con el docente, siendo los MOOC (Massive Open Online Courses) su principal exponente.

Está claro que, en este último escenario, la IA y el BD tendrán un rol cada vez más creciente, pero también es posible que los aprendizajes efectivos en estos entornos sean bastante limitados, como concluye Tony Bates en su libro "Teaching in a Digital Age", "...es necesario que se realice mucha más investigación para identificar qué aprenden realmente los participantes en los MOOC y bajo qué condiciones antes de arribar a conclusiones definitivas" (Bates, 2015, pag. 176).

Sobre el primer escenario antes mencionado, el de la educación tradicional enteramente presencial, cabe mencionar que, si efectivamente no hay involucrada ninguna actividad soportada o viabilizada a través de alguna tecnología digital, difícilmente la IA tenga algo que ofrecer al respecto, ya que no habrá datos sistematizados en plataforma informática alguna que puedan ser procesados automáticamente.

Nos resta entonces analizar el segundo escenario, que es el que debería ser el más utilizado en la actualidad, especialmente en la educación secundaria y universitaria, y es allí donde la IA podría ofrecer algunas oportunidades de aplicación. En efecto, cuanto más utilicemos las tecnologías 
digitales como soporte y/o apoyo de los procesos de enseñanza y aprendizaje, más oportunidades habrá para que los algoritmos inteligentes procesen la información generada por los alumnos y los docentes al utilizar aquellas tecnologías, pero como dijo sabiamente Bates en la cita previa, necesitamos mucha más investigación al respecto. De acuerdo con Oppenheimer, el escenario más probable es aquel en el cual la IA será una herramienta muy útil en las manos de un profesional idóneo que sepa cómo aprovecharla para potenciar sus propios conocimientos y habilidades. (Oppenheimer, 2018)

Al analizar este avance tecnológico, es evidente que hay más preguntas que respuestas, pero de todas formas trataremos de llegar a alguna conclusión que sopese adecuadamente las ventajas y desventajas sin caer en posturas facilistas de rechazo a las innovaciones, actitud que sería lejana a la esencia que pretendemos en los docentes, que sean abiertos al cambio y a las innovaciones en la educación.

\section{Reflexiones finales}

El análisis de la influencia de la IA y el BD en la sociedad contemporánea no se limita únicamente al campo educativo. Así, hoy uno de los sectores más potencialmente afectables por estas tecnologías es el laboral. Según varios estudios, en los próximos años un gran porcentaje de trabajadores podrían verse afectados, o directamente perder su trabajo, como consecuencia de la incorporación de las IA en los ambientes laborales (PwC, s.f.; Frontier Economics, 2018; Burkhardt, 2019).

En el ámbito que nos ocupa, uno de los temores que se menciona frecuentemente cuando se discuten estos temas es si estas tecnologías podrían reemplazar la labor docente. Definitivamente el único docente que podría ser reemplazado por un sistema informático basado en IA, es aquel que solo se limita a "transmitir" conocimientos de una manera mecánica y lineal, y destacamos la palabra "transmitir" porque estamos convencidos que el conocimiento no se transmite como si fueran ondas electromagnéticas que viajan de la mente del profesor a la mente del alumno.

De acuerdo a las palabras de Paulo Freire, "Enseñar no es transferir conocimiento, sino crear las posibilidades para su propia producción o construcción" (Freire, 1997). Todos nosotros conocemos esos profesores para los cuales vale una frase de origen difícil de rastrear, pero que grafica claramente lo que pasa en sus aulas: "Dar clase es ese proceso misterioso mediante el cual los contenidos del cuaderno de notas del profesor se transfieren [...] al cuaderno de notas del estudiante sin pasar por la mente de ninguno de los dos" (Quote Investigator). Aunque resulte difícil para algunos colegas docentes que utilizan este estilo de enseñanza, si son reemplazados por un "profesor artificial", será un beneficio para la sociedad. En efecto, el periodista y escritor Andrés Oppenheimer dice en su libro ¡Sálvese quien pueda! que:

Aunque robots tutores como el Profesor Einstein reemplacen a muchos docentes, harán falta maestros escolares y prescolares para ayudar a los niños a encontrar su vocación y enseñarles habilidades blandas, como la ética, la empatía, el trabajo en equipo, la persistencia y la tolerancia al fracaso. $Y$ harán falta profesores universitarios para atender las necesidades de educación de por vida para cientos de millones de personas. Los robots y las tabletas van a reemplazar muchas de las funciones de transmisión de conocimientos que hoy cumplen los maestros, pero no van a poder ser tan efectivos como los humanos para fomentar la curiosidad de los niños y para mantener permanentemente actualizados y motivados a los adultos. (Oppenheimer, 2018, pag. 321) 
A modo de conclusión, citamos una frase de un libro que ofrece un gran aprendizaje para el mundo que vivimos y el que se aproxima, y los desafíos que enfrentaremos de acuerdo al panorama actual. Se trata de "La Segunda Era De Las Máquinas" (Brynjolfsson y McAfee, 2013) en el cual sus autores postulan que "La tecnología no es el destino. Nosotros moldeamos nuestro destino".

\section{Referencias}

Bates, A. (2015). Teaching in a Digital Age. Pressbooks. https://pressbooks.bcca mpus.ca/teachinginadigitalagev2/

Brynjolfsson, E., y McAfee, A. (2013). La Segunda Era De Las Maquinas. Buenos Aires: Temas.

Burkhardt, M. (14 de abril de 2019). Towards Data Science. Recuperado el 27 de julio de 2019, de https://towardsda tascience.com/the-impact-of-ai-on-ineq uality-job-automation-and-skills-of-the-f uture-fe89c21e34bc

Castro, M., Meier, R., Cukierman, U. y Waigandt, D. (2015). "ICT needs and trends in engineering education", 2015 International Conference on Interactive Collaborative Learning (ICL), Florence, 2015, pp. 146-149, doi: 10.1109/ICL. 2015.7318016 .

Cukierman, U. (2005). AMERICA@UTN learning through advanced communication and information technology resources and means @ UTN. 6th International Conference on Information Technology Based Higher Education and Training, Santo Domingo, 2005, pp. T4C/1-T4C/6, doi: 10.1109/ITHET.2005.1560262.

Cukierman, U., Silvestri, S., Drangosch, J., Pérez Ferrando, D., Agüero, M., Delmonte, R., González Corrao, L. y Saclier, L. (2017). Bridging the gap between first-year students and
Engineering: A novel application of mobile technologies for improving Mathematics and Physics learning. 7th World Engineering Education Forum (WEEF), Kuala Lumpur, 2017, pp. 834-838, doi: 10.1109/WEEF.2017. 8467127.

Cukierman, U., Silvestri, S., Drangosch, J., Pérez Ferrando, D., Agüero, M., González, M., González, C. y Dellepiane, P. (2018). A StudentCentered Approach to Learning Mathematics and Physics in Engineering Freshmen Courses. 2018 World Engineering Education Forum Global Engineering Deans Council (WEEF-GEDC), Albuquerque, NM, USA, pp. 1-5, doi: 10.1109/WEEF -GEDC.2018.8629733.

Deloitte - Ipsos MORI (2019). 2nd Survey of Schools: ICT in Education. Luxemburgo: European Commission, DirectorateGeneral of Communications Networks, Content y Technology.

EdSurge. (2016). Decoding Adaptive. Londres: Pearson.

El Alfy, S., Marx Gómez, J. y Dani, A. (Septiembre de 2018). Exploring the benefits and challenges of learning analytics in higher education institutions: A systematic literature review. Information Discovery and Delivery.

Freire, P. (1997). Pedagogía de la autonomía: Saberes necesarios para la práctica educativa. México: Siglo XXI.

Frontier Economics (2018). THE IMPACT OF ARTIFICIAL INTELLIGENCE ON WORK: An evidence review prepared for the Royal Society and the British Academy. Recuperado el 27 de julio de 2019, de https://royalsociety.org/-/media /policy/projects/ai-and-work/frontier-revi ew-the-impact-of-Al-on-work.pdf

Gardner, H. (1991). The unschooled mind: how children think and how schools should teach. New York: Basic Books. 
Gardner, H., y Hatch, T. (1989). Multiple intelligences go to school: Educational implications of the theory of multiple intelligences. Educational Researcher, 18(8), págs. 4-9.

Gardner, H. (1983). Frames of mind: the theory of multiple intelligences. New York: Basic Books.

Grigori, D., Casati, F., Castellanos, M., Dayal, U., Sayal, M. y Shan, M. (2004). Business Process Intelligence. Computers in Industry, 53, págs. 321-343.

Halevi, G. y Moed, H. (2012). The Evolution of Big Data as a Research and Scientific Topic: Overview of the Literature. Research Trends, 30, 3-6. Recuperado el 22 de julio de 2019, de https://www.researchtrends.com/issue30-september-2012/the-evolution-of-big -data-as-a-research-and-scientific-topicoverview-of-the-literature/

International Telecommunications Union (2019). World Telecommunication/ICT Indicators Database 2018.

McCarthy, J., Minsky, M., Rochester, N. y Shannon, C. (1955). A Proposal for the Dartmouth Summer Research Project on Artificial Intelligence. Recuperado el 22 de julio de 2019, de http://jmc.stanford.edu/articles/dartmout h/dartmouth.pdf

New Media Consortium (2016). 2016 Horizon Report. EDUCAUSE. Obtenido de https://library.educause.edu/-/media /files/library/2016/2/2016hrhees.pdf

Nunn, S., Avella, J., Kanai, T. y Kebritchi, M. (junio de 2016). Learning Analytics Methods, Benefits, and Challenges in
Higher Education: A Systematic Literature Review. Online Learning Journal, 20(2), págs. 1-17.

Oppenheimer, A. (2018). iSálvese quien pueda! El futuro del trabajo en la era de la automatización. México: Debate.

Perry, S. (22 de mayo de 2017). What is big data? More than volume, velocity and variety.... Recuperado el 22 de julio de 2019, de https://developer.ibm.com /dwblog/2017/what-is-big-data-insight/

PwC (s.f.). PwC - UK. Recuperado el 27 de julio de 2019, de https://www.pwc.co.uk /services/economics-policy/insights/theimpact-of-automation-on-jobs.html

Quote Investigator (s.f.). Recuperado el 27 de julio de 2019, de https://quoteinvestigator.com/2012/08/1 7/lecture-minds/\#return-note-4284-2

Real Academia Española (s.f.). Diccionario de la Lengua Española. Recuperado el 22 de julio de 2019, de https://dle.rae.es/?id=LqtyoaQ

Technavio (2018). Artificial Intelligence Market in the US Education Sector 2018-2022. Londres: Technavio.

UNESCO (2012). UNESCO Institute for Statistics. Recuperado el 26 de julio de 2019, de http://data.uis.unesco.org/

Web Analytics Association (16 de agosto de 2007). Web Analytics Definitions. Recuperado el 23 de julio de 2019, de http://www.inesting.org/ad2006/adminsc 1/app/marketingtecnologico/uploads/Ma nuais/waa-standards-analytics-definitio ns-volume-i-20070816.pdf 